\title{
On circulant thin Lehman matrices
}

\author{
Tadashi Sakuma • Hidehiro Shinohara
}

Received: 11 September 2011 / Accepted: 16 February 2014 / Published online: 29 March 2014

(C) Springer Science+Business Media New York 2014

\begin{abstract}
The class of Lehman matrices is a key structure of characterization of minimally non-ideal clutters. The notion of 1-overlapped factorizations of cyclic groups produces an infinite family of circulant thin Lehman matrices. In this paper, we prove essential properties of the 1-overlapped factorizations of cyclic groups and completely determine the shapes of circulant thin Lehman matrices with small constant line sum, which solves the ideal counterpart of the so-called Grinstead's conjecture for the circulant partitionable graphs in this case.
\end{abstract}

Keywords Thin Lehman matrix $\cdot 1$-Overlapped factorization · Cyclic group

\section{Introduction}

\subsection{Definitions and notation}

An $n \times n$ matrix $X$ is called a Lehman matrix if there exists an $n \times n$ matrix $Y$ and a positive integer $d$ such that $X Y^{T}=J_{n}+d I_{n}$, where $J_{n}$ is the $n \times n$ all-one matrix and $I_{n}$ is the identity matrix. A pair of Lehman matrices $X, Y$ is thin if $d=1$ and fat otherwise. On the other hand, it is well known that a partitionable graph has a

T. Sakuma

Systems Science and Information Studies, Faculty of Education, Art and Science, Yamagata University, 1-4-12 Kojirakawa, Yamagata 990-8560, Japan

e-mail: sakuma@e.yamagata-u.ac.jp

H. Shinohara $(\varangle)$

Division of Mathematics, Graduate School of Information Sciences, Tohoku University, 6-3-09

Aramaki-Aza-Aoba, Aoba-ku, Sendai 980-8579, Japan

e-mail: shinohara@math.is.tohoku.ac.jp 
maximum clique matrix $X$ and a maximum stable set matrix $Y$ such that $X Y=J_{n}-I_{n}$ (see the definition of partitionable graphs in [12]).

We say that a matrix $M$ has a constant line sum if there exists an integer $r$ such that each row and column sum of $X$ is $r$. The following theorem is essential to study Lehman matrices and clique matrices of partitionable graphs.

Theorem 1.1 (Bridges and Ryser [1]) Let $\lambda, \mu$ be relatively prime integers and suppose that $\lambda I_{n}+\mu J_{n}=X Y$, where $X$ and $Y$ are $n \times n$ nonnegative integral matrices. Then, $X$ and $Y$ have constant line sums.

From this theorem, a Lehman matrix or a maximum clique matrix of a partitionable graph has a constant line sum. An $n \times n$-matrix $A=\left[a_{i, j}\right]$ is circulant if $a_{i, j}=$ $a_{i+1, j+1}$, where the indices are read modulo $n$. The aim of this paper is to characterize the shapes of circulant thin Lehman matrices with small line sums.

\subsection{Motivations}

In this subsection, $\mathscr{V}=V(\mathscr{H})$ denotes a set of $n$ elements and $\mathscr{H}=(\mathscr{V}, \mathscr{E})$ denotes a hypergraph on $\mathscr{V}$ with hyperedge set $\mathscr{E}=E(\mathscr{H})$. A hypergraph $\mathscr{H}=(\mathscr{V}, \mathscr{E})$ is a clutter if $E_{1} \subseteq E_{2}$ yields $E_{1}=E_{2}$ for each $E_{1}, E_{2} \in \mathscr{E}$. The blocker $b(\mathscr{H})$ of a clutter $\mathscr{H}$ is a clutter such that its vertex set is $\mathscr{V}$ and its hyperedge set consists of minimal members of $\{B \subseteq \mathscr{V}|| B \cap A \mid \geq 1$ for all $A \in \mathscr{E}\}$. A representation matrix $M(\mathscr{H})$ of a clutter $\mathscr{H}$ is a $0-1$ matrix with rows indexed by $\mathscr{E}$; and columns indexed by $\mathscr{V}$ such that the $(i, j)$-th entry of $X$ equals to 1 if $V_{j} \in E_{i}$, where $V_{j} \in \mathscr{V}$ and $E_{i} \in \mathscr{E}$.

In this paper, we identify a clutter with its representation matrix. A representation matrix of $M(b(\mathscr{H}))$ is also called a blocker of $M(\mathscr{H})$.

We say that a clutter $\mathscr{H}$ or its representation matrix is an ideal clutter or an ideal matrix if its blocking polyhedron

$$
\{\vec{x} \in \mathbb{R} \mid \overrightarrow{0} \leq \vec{x}, M(\mathscr{H}) \vec{x} \geq \overrightarrow{1}\}
$$

is integral (in other words, it coincides with its set covering polyhedron). For a vertex $j \in V(\mathscr{H})$, deletion $\mathscr{H} \backslash j$ and contraction $\mathscr{H} / j$ are clutters defined as follows.

- $V(\mathscr{H} \backslash j)=\mathscr{V} \backslash j, E(\mathscr{H} \backslash j)=\left\{E_{l} \in \mathscr{E} \mid j \notin E_{l}\right\}$.

- $V(\mathscr{H} / j)=\mathscr{V} \backslash j, E(\mathscr{H} / j)$ is the minimal members of $\left\{E_{l}-\{j\} \mid E_{l} \in \mathscr{E}\right\}$.

A minor of $\mathscr{H}$ is a clutter which is obtained from $\mathscr{H}$ by repeatedly taking deletions and contractions. Seymour [17] proved that these two operations preserve the idealness of a clutter. A clutter or its representation matrix is minimally non-ideal if it is not an ideal matrix but all its minors are.

We say that a matrix $X_{1}$ is isomorphic to $X_{2}$ if there exists a pair of permutation matrices $P_{1}$ and $P_{2}$ such that $X_{1}=P_{1} X_{2} P_{2}$. A degenerate projective plane $\mathscr{J}_{n}$ is an $n \times n$ 0-1 matrix such that $(i, j)$-th entry equals 1 , if $n \geq 3$ and one of the following hold; (i) $i=1$ and $j \geq 2$, (ii) $i \geq 2$ and $j=1$, and (iii) $i=j \geq 2$. In 1979, A. Lehman proved the following property of minimally non-ideal matrices. 
Theorem 1.2 (Lehman [13]) A minimally non-ideal matrix $X$ and its blocker $Y$ satisfy one of the following.

- Both $X$ and $Y$ are isomorphic to a degenerate projective plane $\mathscr{J}_{n}$ for some $n \geq 3$. - $X$ and $Y$ are isomorphic to $\left[\begin{array}{l}X_{1} \\ X_{2}\end{array}\right]$ and $\left[\begin{array}{l}Y_{1} \\ Y_{2}\end{array}\right]$ respectively, where $X_{1}$ and $Y_{1}$ are square matrices with $X_{1} Y_{1}^{T}=J_{n}+d I_{n}$ for some positive integer $d$ and each row of $X_{2}$ (resp. $Y_{2}$ ) has more 1 s than the constant line sum of $X_{1}$ (resp. $\left.Y_{1}\right)$.

Perfection is another important concept in clutter theory. A clutter is called perfect if its anti-blocking polytope

$$
\left\{\vec{x} \in \mathbb{R}^{n} \mid \overrightarrow{0} \leq \vec{x}, M(\mathscr{H}) \vec{x} \leq \overrightarrow{1}\right\}
$$

is integral (in other words, it coincides with its set packing polytope). Sachs [16] proved that a clutter is perfect if and only if it is a clique clutter of a perfect graph. A graph is partitionable if its maximum clique matrix $X$ and its maximum stable set matrix $Y$ satisfy $X Y^{T}=J-I$. Since Padberg [15] proved that each minimally imperfect graph is partitionable, this matrix equation has been well studied. Many researchers have discovered essential similarities between properties of Lehman matrices and of the clique matrices of partitionable graphs (see $[9,10,18]$, etc).

However, as Shepherd pointed out in [18], the proofs of the theorems concerned with the Lehman (ideal) matrices are usually more difficult and complicated than the partitionable (perfect) counterparts. The first and most importantly, in the case of the anti-blocking polytope of a clutter matrix, its integrality (perfectness) and the totally dual integrality of its linear system coincide. (A matrix inequality $A \vec{x} \geq \vec{b}$ (resp. $A \vec{x} \leq \vec{b})$ is called totally dual integral if the linear program $\min \{\langle\vec{w}, \vec{x}\rangle \mid A \vec{x} \geq \vec{b}\}$ (resp. $\max \{\langle\vec{w}, \vec{x}\rangle \mid A \vec{x} \leq \vec{b}\}$ ) has an integral optimal dual solution $\vec{y}$ for every integral cost vector $\vec{w}$ for which the above linear program has a finite optimum.) On the other hand, there exists a significant gap between the integrality (ideality) and the totally dual integrality (Mengerian Property) of the blocking polyhedra of clutter matrices. For more details, consult Cornuéjols [4]. Secondly, due to the Strong Perfect Graph Theorem [2], the clique matrices of the minimally imperfect graphs turn to be the only special circulant matrices $C_{2}^{2 n+1}$ and $C_{n}^{2 n+1}$ s. However, Wang [23] disproved a conjecture proposed by Cornuéjols and Novick [6] which would have been a counterpart of the Strong Perfect Graph Theorem, and discovered an infinite family of minimally non-ideal matrices which are not circulant. Cornuéjols et al. [5] studied thin Lehman matrices by using Kolmogorov complexity. However, the characterization of minimally non-ideal matrices seems completely out of reach, even if we restrict ourselves to the case of thin Lehman matrices.

Despite all such difficulties, the authors expect that there are essential similarities between the circulant thin Lehman matrices and the clique matrices of the circulant partitionable graphs, which may give the complete characterizations for both sets of matrices at the same time. 


\subsection{1-Overlapped factorizations and near-factorizations}

For two subsets $S_{1}, S_{2}$ of an abelian group $G$, let $S_{1}+S_{2}$ denote the multiset $\{a+b \mid$ $\left.a \in S_{1}, b \in S_{2}\right\}$. For a subset $S \subset G$ and an element $p \in G$, we use the abbreviation $S+p$ to denote the set $S+\{p\}$. A subset $S \subset G$ is symmetric if $S=-S$, and shift-symmetric if there exists an element $g \in G$ such that $S+g=-S$.

A pair of subsets $(A, B)$ of an abelian group $G$ is called a 1-overlapped factorization if the multiset $A+B$ equals $G \cup\{g\}$ for some $g \in G$ and $|A|,|B| \geq 2$ hold. In this case, $(A, B)$ satisfies $|A| \cdot|B|=|G|+1$. The unique element with two expression is called the doubly covered element of a 1-overlapped factorization $(A, B)$. For a subset $S \subset G$, let $M(S)$ be the matrix such that its each rows and columns are indexed by the elements of $G$ and the $(i, j)$-th entry is 1 if $g_{i}^{-1} g_{j} \in S$, and 0 otherwise. For a 1-overlapped factorization $(A, B)$, we have $M(A) M(B)=J_{n}+I_{n}$. On the other hand, Chvátal et al. [3] introduced the notion of near-factorizations of finite groups; that is, a pair of $(A, B)$ satisfies $|A B|=|A| \cdot|B|=|G|-1$ and $|A|,|B| \geq 2$. A pair $(A, B)$ of subsets of a finite group $G$ is a factorization if it satisfies $|A B|=|A| \cdot|B|=|G|$ and $|A|,|B| \geq 2$. A 1-overlapped factorization $(A, B)$ of $G$ is a symmetric 1-overlapped factorization if both of $A$ and $B$ are symmetric, and the doubly covered element is 0 . Near-factorizations and 1-overlapped factorizations are interesting objects by themselves, since they are close to the factorizations of finite groups and they have some surprising properties where factorizations do not have (see $[8,19,21]$ ).

In [19], one of the authors constructed 1-overlapped factorizations of finite cyclic groups by Degenerate British Number System (DBNS for short) which was originally studied by De Bruijn [7]. For integers $l$ and $m$, let $[l, m]$ denote the set of integers from $l$ to $m$. Let $\varphi_{k}$ be an homomorphism from $\mathbb{Z}$ to $\mathbb{Z}_{k}$ such that $\varphi_{k}(i)$ is the residue of $i$ modulo $k$. We sometime use $\varphi$ instead of $\varphi_{n}$. Let $\rho(\geq 1)$ and $m_{1}, m_{2}, \ldots, m_{2 \rho}(\geq 2)$, be integers such that $r=\prod_{i=1}^{\rho} m_{2 i-1}, s=\prod_{i=1}^{\rho} m_{2 i}$ and $n=\prod_{i=1}^{2 \rho} m_{i}$. Let $\mu_{j}=\prod_{i=1}^{j-1} m_{i}$ for $2 \leq j \leq 2 \rho$ and $\mu_{1}=1$. Define a subset $M_{i}$ of $\mathbb{N}$ by $\left[0, m_{i}-1\right] \mu_{i}, A^{\prime}:=M_{1}+M_{3}+\cdots+M_{2 \rho-1}$ and $B^{\prime}:=M_{2}+M_{4}+\cdots+M_{2 \rho}$. It is clear that $(A, B):=\left(\varphi_{n-1}\left(A^{\prime}\right), \varphi_{n-1}\left(B^{\prime}\right)\right)$ is a 1-overlapped factorization of $\mathbb{Z}_{n-1},\left(\varphi_{n+1}\left(A^{\prime}\right), \varphi_{n+1}\left(B^{\prime}\right)\right)$ is a near-factorization of $\mathbb{Z}_{n+1}$ and $\left(\varphi_{n}(A), \varphi_{n}(B)\right)$ is a factorization of $\mathbb{Z}_{n}$ with parameters $\left(m_{1}, m_{2}, \ldots, m_{2 \rho}\right.$. We say that this $(A, B)$ is a basic DBNS 1-overlapped factorization (resp. a basic DBNS near-factorization, a basic DBNS factorization) of $\mathbb{Z}_{n}$. For this 1-overlapped factorization (resp. nearfactorization and factorization), the following three operations construct other 1overlapped factorizations (resp. near-factorizations and factorizations);

- Shifting $(A+a, B+b)$ for some $a, b \in \mathbb{Z}_{n}$,

- Scaling $(\lambda A, \lambda B)$ for some $\lambda \in \mathbb{Z}_{n}^{\times}$,

- Swapping $(-A, B)$.

We say a 1-overlapped factorization (resp. a near-factorization and a factorization) constructed by this method is a DBNS 1-overlapped factorization (resp. a DBNS nearfactorization, a factorization) of $\mathbb{Z}_{n}$. A Lehman matrix $X$ is called a DBNS Lehman matrix (resp. a DBNS partitionable graph) if there exists a DBNS 1-overlapped factorization $(A, B)$ (resp. DBNS near-factorization $(A, B))$ such that $X$ is isomorphic to $M(A)$. Here, we prove that $M(B)$ is also a DBNS Lehman matrix. 
Lemma 1.3 Let $(A, B)$ be a DBNS 1-overlapped factorization. Then, $M(B)$ is a DBNS Lehman matrix.

Proof Note that $\left(\left(\varphi\left(m_{1}\right)\right)^{-1} B,\left(\varphi\left(m_{1}\right)\right)^{-1} A\right)$ is a DBNS 1-overlapped factorization with $2 \rho$ parameters $m_{2}, m_{3}, \ldots, m_{2 \rho-1}, m_{2 \rho}, m_{1}$. Moreover, we can obtain $\left(\left(\varphi\left(m_{1}\right)\right)^{-1} A,\left(\varphi\left(m_{1}\right)\right)^{-1} B\right)$ from $(A, B)$ by a scaling. Since $M(B)$ is isomorphic to a DBNS Lehman matrix $M\left(\left(\varphi\left(m_{1}\right)\right)^{-1} B\right), B$ is also a DBNS Lehman matrix.

\subsection{Grinstead's conjecture and Lehman matrix counterpart}

In 1984, Grinstead [11] proposed the following structural conjecture on circular partitionable graphs.

Conjecture 1.4 All near-factorizations of finite cyclic group are DBNS nearfactorizations.

It is well known that this conjecture is an algebraic approach to the strong perfect graph theorem, and it is an important problem after settlement of the strong perfect graph theorem. Kashiwabara and one of the authors [12] proved that Grinstead's conjecture holds if $\min (|A|,|B|) \leq 8$. As for the Lehman matrix counterpart of Grinstead's conjecture, we have naturally the following conjecture.

Conjecture 1.5 All 1-overlapped factorizations of finite cyclic groups are DBNS 1overlapped factorizations.

In the recent paper [22], Wang proposed an inadequate conjecture which argues that every circulant thin Lehman matrix is a DBNS Lehman matrix with rank at most two. Conjecture 1.5 indicates that the patterns of circulant thin Lehman matrices should have richer (but still moderate) structure than the expectation set by Wang. As a main result of this paper, we will prove that Conjecture 1.5 holds if $\min (|A|,|B|) \leq 8$.

One may argue that one should take account of the cases of $m_{i}=1$. In Sect. 2, we prove that the condition $m_{i}=1$ does not change the definition of DBNS 1-overlapped factorizations.

\section{Preliminaries}

The following theorems are useful for studying 1-overlapped factorizations.

Theorem 2.1 (Shinohara [19]) Let $(A, B)$ be a 1-overlapped factorization of a finite group $G$. Then, we have $\langle A\rangle=\langle B\rangle=G$.

Theorem 2.2 (Shinohara [19]) Let $(A, B)$ be a 1-overlapped factorization of a finite abelian group $G$. Then, there exist two elements $a, b \in G$ such that $(A+a, B+b)$ is a symmetric 1-overlapped factorization of $G$.

Here, we prove that there are no "new" 1-overlapped factorizations of $\mathbb{Z}_{n}$ even if we change the definition of parameters from $m_{i} \geq 2$ to $m_{i} \geq 1$. We say that a 1overlapped factorization $(A, B)$ of $\mathbb{Z}_{n}$ is a skew 1-overlapped factorization if there 
exist $2 \rho$ integers $m_{1}, m_{2}, \ldots, m_{2} \rho$ such that there exists an index $i$ with $m_{i}=1$ and that

$$
A=\sum_{j=1}^{\rho}\left[0, m_{2 j-1}-1\right] \prod_{k=1}^{2 j-2} m_{k}, \quad B=\sum_{j=1}^{\rho}\left[0, m_{2 j}-1\right] \prod_{k=1}^{2 j-1} m_{k}
$$

If $\rho=1$, then we have $\min \{|A|,|B|\}=1$, a contradiction. Hence, a skew 1-overlapped factorization satisfies $\rho \geq 2$.

Lemma 2.3 Let $(A, B)$ be a skew 1-overlapped factorization with parameters $m_{1}, m_{2}, \ldots, m_{2} \rho$. If there exists a parameter $m_{i}=1$ such that $2 \leq i \leq 2 \rho-1$, then $(A, B)$ is a basic DBNS or skew 1-overlapped factorization with $2 \rho-2$ parameters $m_{1}, m_{2}, \ldots, m_{i-2}, m_{i-1} m_{i+1}, m_{i+2}, \ldots, m_{2 \rho}$.

Proof Let $M_{j}$ denote the set $\left[0, m_{j}-1\right] \prod_{k=1}^{j-1} m_{k}$ for $1 \leq j \leq 2 \rho$, and $M_{j}^{\prime}$ be the set defined by as follows;

$$
M_{j}^{\prime}:= \begin{cases}{\left[0, m_{j}-1\right] \prod_{k=1}^{j-1} m_{k}} & (\text { if } 1 \leq j \leq i-2), \\ {\left[0, m_{j} m_{j+2}-1\right] \prod_{k=1}^{j-1} m_{k}} & (\text { if } j=i-1), \\ {\left[0, m_{j+2}-1\right] \prod_{k=1}^{j+1} m_{k}} & (\text { if } i \leq j \leq 2 \rho-2)\end{cases}
$$

Let $A^{\prime}, B^{\prime}$ denote the set

$$
A^{\prime}=\sum_{k=1}^{\rho-1} M_{2 k-1}, \quad B^{\prime}=\sum_{k=1}^{\rho-1} M_{2 k}
$$

Clearly, we have

$$
M_{j}^{\prime}= \begin{cases}M_{j} & (\text { if } 1 \leq j \leq i-2) \\ M_{j+2} & (\text { if } i \leq j \leq 2 \rho-2)\end{cases}
$$

Moreover, we have

$$
\begin{aligned}
M_{i-1}+M_{i+1} & =\left[0, m_{i-1}-1\right] \prod_{k=1}^{i-2} m_{k}+\left[0, m_{i+1}-1\right] \prod_{k=1}^{i} m_{k} \\
& =\left(\left[0, m_{i-1}-1\right]+\left[0, m_{i+1}-1\right] m_{i-1}\right) \prod_{k=1}^{i} m_{k} \\
& =\left[0, m_{i-1} m_{i+1}-1\right] \prod_{k=1}^{i} m_{k} \\
& =M_{i-1}^{\prime}
\end{aligned}
$$

Therefore, we have $A=A^{\prime}$ and $B=B^{\prime}$. 
Thus, the definition of DBNS 1-overlapped factorization does not change even if we remove the restriction $m_{i} \neq 1$ for $2 \leq i \leq 2 \rho-1$. We prove that the cases $m_{1}=1$ and $m_{2 \rho}=1$ do not change the definition of DBNS 1-overlapped factorization.

Lemma 2.4 Let $(A, B)$ be a skew 1-overlapped factorization with $2 \rho$ parameters $m_{1}$, $m_{2}, m_{3}, \ldots, m_{2 \rho-1}, m_{2 \rho}$. If $m_{2}, m_{3}, \ldots, m_{2 \rho-1}$ are greater than $1, m_{2 \rho} \geq 1$, and $m_{1}=1$, then

$$
\left(\left(\varphi\left(m_{2}\right)\right)^{-1} A,\left(\varphi\left(m_{2}\right)\right)^{-1} B\right)
$$

is a DBNS 1-overlapped factorization with $2 \rho-2$ parameters $m_{3}, m_{4}, \ldots$, $m_{2 \rho-1}, m_{2 \rho} m_{2}$.

Proof Since we have $\prod_{k=1}^{2 \rho} m_{2 k}=n+1, \varphi\left(m_{2}\right)$ is an invertible element of $\mathbb{Z}_{n}$. Let $M_{j}$ be the set $\left[0, m_{j}-1\right] \prod_{k=1}^{j-1} m_{k}(1 \leq j \leq 2 \rho)$. Define $m_{j}^{\prime}:=m_{j+2}$ for $1 \leq j \leq 2 \rho-3$, and $m_{2 \rho-2}^{\prime}:=m_{2} m_{2 \rho}$. Let $M_{j}^{\prime}$ be the set $\left[0, m_{j}^{\prime}-1\right] \prod_{k=1}^{j-1} m_{k}^{\prime}$. Then we have

$$
\begin{aligned}
\left(\varphi\left(m_{2}\right)\right)^{-1} \varphi\left(M_{j+2}\right) & =\varphi\left(\left[0, m_{j+2}-1\right]\right)\left(\varphi\left(m_{2}\right)\right)^{-1} \varphi\left(\prod_{k=1}^{j+1} m_{k}\right) \\
& =\varphi\left(\left[0, m_{j+2}-1\right]\right) \varphi\left(\prod_{k=3}^{j+1} m_{k}\right) \\
& =\varphi\left(\left[0, m_{j}^{\prime}-1\right] \prod_{k=1}^{j-1} m_{j}^{\prime}\right) \\
& =\varphi\left(M_{j}^{\prime}\right) .
\end{aligned}
$$

Moreover, we have

$$
\begin{aligned}
& \left(\varphi\left(m_{2}\right)\right)^{-1}\left(\varphi\left(M_{2}+M_{2 \rho}\right)\right) \\
& \left.=\varphi\left(\left[0, m_{2}-1\right]\right)\left(\varphi\left(m_{2}\right)\right)^{-1}\right)\left(\varphi\left(m_{1}\right)\right)+\varphi\left(\left[0, m_{2 \rho}-1\right]\right)\left(\varphi\left(m_{2}\right)^{-1}\right) \varphi\left(\prod_{k=1}^{2 \rho-1} m_{k}\right) \\
& =\varphi\left(\left[0, m_{2}-1\right]\right) \varphi\left(\prod_{k=3}^{2 \rho} m_{k}\right) \varphi(1)+\varphi\left(\left[0, m_{2 \rho}-1\right]\right) \varphi\left(\prod_{k=3}^{2 \rho-1} m_{k}\right) \\
& =\left(\varphi\left(\left[0, m_{2}-1\right]\right) m_{2 \rho}+\varphi\left(\left[0, m_{2 \rho}-1\right]\right)\right) \varphi\left(\prod_{k=3}^{2 \rho-1} m_{k}\right) \\
& =\varphi\left(\left[0, m_{2} m_{2 \rho}-1\right]\right) \varphi\left(\prod_{k=1}^{2 \rho-3} m_{k}^{\prime}\right) \\
& =\varphi\left(M_{2 \rho-2}^{\prime}\right)
\end{aligned}
$$


Thus, we have $\left(\varphi\left(m_{2}\right)\right)^{-1} \varphi(A)=\varphi\left(A^{\prime}\right)$ and $\left(\varphi\left(m_{2}\right)\right)^{-1} \varphi(B)=\varphi\left(B^{\prime}\right)$.

Lemma 2.5 Let $(A, B)$ be a skew 1-overlapped factorization with $2 \rho$ parameters $m_{1}, m_{2}, \ldots, m_{2 \rho}$. If $m_{2}, \ldots, m_{2 \rho-1}$ are greater than $1, m_{1} \geq 1$, and $m_{2 \rho}=1$, then $\left(\varphi\left(m_{2 \rho-1}\right) A, \varphi\left(m_{2 \rho-1}\right) B\right)$ is a basic DBNS 1-overlapped factorization with $2 \rho-2$ parameters $m_{1} m_{2 \rho-1}, m_{2}, m_{3}, \ldots, m_{2 \rho-2}$.

Proof Let $M_{j}$ be the set $\left[0, m_{j}-1\right] \prod_{k=1}^{j-1} m_{k}$. Define $m_{j}^{\prime}:=m_{j}$ for $2 \leq j \leq 2 \rho-2$, and $m_{1}^{\prime}:=m_{1} m_{2 \rho-1}$. Let $M_{j}^{\prime}$ be the set $\left[0, m_{j}^{\prime}-1\right] \prod_{k=1}^{j-1} m_{k}^{\prime}$, and $A^{\prime}$ and $B^{\prime}$ the set $\sum_{j=1}^{\rho-1} M_{2 j-1}, \sum_{j=1}^{\rho-1} M_{2 j}$, respectively. For $2 \leq j \leq 2 \rho-2$, we have

$$
\begin{aligned}
\varphi\left(m_{2 \rho-1}\right) \varphi\left(M_{j}\right) & =\varphi\left(m_{2 \rho-1}\right) \varphi\left(\left[0, m_{j}-1\right]\right) \varphi\left(\prod_{k=1}^{j-1} m_{k}\right) \\
& =\varphi\left(\left[0, m_{j}-1\right]\right) \varphi\left(m_{2 \rho-1} m_{1}\right) \varphi\left(\prod_{k=2}^{j-1} m_{k}\right) \\
& =\varphi\left(\left[0, m_{j}^{\prime}-1\right]\right) \varphi\left(\prod_{k=1}^{j-1} m_{k}^{\prime}\right) \\
& =\varphi\left(M_{j}^{\prime}\right) .
\end{aligned}
$$

Moreover, we have

$$
\begin{aligned}
\varphi & \left(m_{2 \rho-1}\right) \varphi\left(M_{1}+M_{2 \rho-1}\right) \\
& =\varphi\left(m_{2 \rho-1}\right)\left(\varphi\left(\left[0, m_{1}-1\right]\right)+\varphi\left(\left[0, m_{2 \rho-1-1}\right]\right) \varphi\left(\prod_{k=1}^{2 \rho-2} m_{k}\right)\right) \\
& =\varphi\left(m_{2 \rho-1}\right) \varphi\left(\left[0, m_{1}-1\right]\right)+\varphi\left(\left[0, m_{2 \rho-1}-1\right]\right) \varphi(n+1) \\
& =\varphi\left(\left[0, m_{1} m_{2 \rho-1}-1\right]\right) \\
& =\varphi\left(M_{1}^{\prime}\right) .
\end{aligned}
$$

Hence, we have $\varphi\left(m_{2 \rho-1}\right) \varphi(A)=\varphi\left(A^{\prime}\right)$ and $\varphi\left(m_{2 \rho-1}\right) \varphi(B)=\varphi\left(B^{\prime}\right)$.

Combining the above three lemmas, we have the following.

Corollary 2.6 A skew 1-overlapped factorization $(A, B)$ is a DBNS 1-overlapped factorization.

\section{Properties of DBNS 1-overlapped factorizations}

In this section, we prove some lemmas characterizing DBNS 1-overlapped factorizations which help us to prove our main theorem. 
Let $(A, B)$ be a 1-overlapped factorization of $\mathbb{Z}_{n}$ with doubly covered element 0 . From Theorem 2.2, we assume that both of $A$ and $B$ are symmetric. Then, we have

$$
\begin{aligned}
|(A+c) \cap B| & =|\{(a, b) \mid a \in A, b \in B, a+c=b\}| \\
& =|\{(a, b) \mid a \in A, b \in B, a+c=-b\}| \quad(\text { since } B=-B) \\
& =|\{(a, b) \mid a \in A, b \in B, a+b=-c\}| \\
& =1+\delta_{0,-c} \\
& =1+\delta_{0, c},
\end{aligned}
$$

where $\delta_{i, j}$ is the Kronecker's delta.

Lemma 3.1 Let $(A, B)$ be a symmetric 1-overlapped factorization of $\mathbb{Z}_{n}$ with doubly covered element 0 . Then, there exists a unique nonzero element $x \in A \cap B$ up to sign such that

$$
\begin{aligned}
(A-A) \cap(B-B) & =\{0, \pm 2 x\}, \\
4 x \neq 0 . &
\end{aligned}
$$

Moreover, if $a_{1}, a_{2} \in A$ (resp. $\left.b_{1}-b_{2} \in B\right)$ satisfy $a_{1}-a_{2}=2 x\left(\right.$ resp. $\left.b_{1}-b_{2}=2 x\right)$, then we have $a_{1}=x, a_{2}=-x$ (resp. $\left.b_{1}=x, b_{2}=-x\right)$.

Proof Since 0 is the doubly covered element of $(A, B)$, there exist two distinct element $x_{1}, x_{2}$ such that

$$
\begin{aligned}
0=x_{1}+\left(-x_{1}\right) & \left(x_{1} \in A,-x_{1} \in B\right), \\
& =x_{2}+\left(-x_{2}\right) \quad\left(x_{2} \in A,-x_{2} \in B\right) .
\end{aligned}
$$

Then, we have $\left\{ \pm\left(x_{1}-x_{2}\right)\right\} \subset((A-A) \cap(B-B)) \backslash\{0\}$.

Next, we show that $|((A-A) \cap(B-B)) \backslash\{0\}| \leq 2$. Let $\left(a_{1}, a_{2}\right)$ and $\left(a_{3}, a_{4}\right)$ be pairs of distinct elements of $A$. Let $b_{1}, b_{2}, b_{3}, b_{4}$ be elements of $B$ such that $a_{1}-a_{2}=b_{1}-b_{2}$, $a_{3}-a_{4}=b_{3}-b_{4}$. Then, $a_{1}+b_{2}=a_{2}+b_{3}$ and $a_{3}+b_{4}=a_{4}+b_{3}$ are doubly covered elements of $(A, B)$. From uniqueness of doubly covered element of $(A, B)$, we obtain $\left\{a_{1}, a_{2}\right\}=\left\{a_{3}, a_{4}\right\}$. Moreover, we have $a_{1}=-b_{2}$ and $a_{2}=-b_{1}$, i.e.,

$$
\left\{ \pm a_{1}, \pm a_{2}\right\} \subset A \cap B
$$

Hence we obtain

$$
\left\{ \pm\left(a_{1}-a_{2}\right)\right\} \supset((A-A) \cap(B-B)) \backslash\{0\} .
$$

Suppose that $((A-A) \cap(B-B)) \backslash\{0\}$ has just one element. Since $a_{1}$ and $a_{2}$ are distinct elements, we have

$$
a_{1}-a_{2}=a_{2}-a_{1}=n / 2 .
$$


Hence $n$ is even. Since $a_{1}-\left(-a_{1}\right)$ belongs to $(A-A) \cap(B-B), 2 a_{1}$ equals one of 0 and $n / 2$.

(Case 1) $2 a_{1}=0$ : In this case, $A \backslash\left\{a_{1}, a_{1}+2 / n\right\}$ has no involutions, i.e., it contains even elements since $A$ is symmetric. Thus, $|A|$ is even. However, this contradicts the fact $n=|A||B|-1$ is even. Thus, $2 a_{1}=0$ yields $|((A-A) \cap(B-B)) \backslash\{0\}| \geq 2$.

(Case 2) $2 a_{1}=n / 2$ : If $A$ or $B$ contains an involution, $|A||B|$ is even, and hence $n$ is odd. Then, both of $A$ and $B$ must contain involutions. However, at the same time $(A-A) \cap(B-B)$ must contain $\{n / 2, \pm n / 4\}$. Consequently, $2 a_{1}=n / 2$ also yields $|((A-A) \cap(B-B)) \backslash\{0\}| \geq 3$, which contradicts (3.5). Thus, the assumption $a_{1}-a_{2}=n / 2$ is false.

Corollary 3.2 Let $(A, B)$ be a 1-overlapped factorization of $\mathbb{Z}_{n}$. Then, there exists an nonzero element $g$ such that

$$
\begin{array}{r}
(A-A) \cap(B-B)=\{0, \pm g\}, \\
2 g \neq 0 .
\end{array}
$$

Moreover, there exists a unique quadruple $\left(a_{1}, a_{2}, b_{1}, b_{2}\right)$ such that $a_{1}, a_{2} \in A$, $b_{1}, b_{2} \in B$ and $a_{1}-a_{2}=b_{1}-b_{2}=g$.

Proof This corollary follows from Theorem 2.2 since shifting does not change the elements of $(A-A) \cap(B-B)$.

Let $\psi_{n}$ denote a map from $\mathbb{Z}_{n}$ to $[0, n-1]$ such that $\psi_{n} \circ \varphi_{n}(i)=i$ for each $i \in[0, n-1]$.

Lemma 3.3 (De Bruijn [7]) Let $A^{\prime}$ and $B^{\prime}$ be subsets of $\mathbb{Z}_{n}$ such that $\psi_{n}\left(A^{\prime}+B^{\prime}\right)=$ $[0, x-1]$ and $\left|A^{\prime}\right| \cdot\left|B^{\prime}\right|=x$ for some $x \in[0, n]$. Then, there exist natural numbers $\rho^{\prime}(\geq 1), m_{2}^{\prime}, \ldots, m_{2 \rho^{\prime}-1}^{\prime}(\geq 2), m_{1}^{\prime}, m_{2 \rho^{\prime}}^{\prime}(\geq 1)$ such that $\prod_{k=1}^{2 \rho^{\prime}} m_{k}=x$, $A^{\prime}=\varphi\left(\sum_{j=1}^{\rho^{\prime}}\left[0, m_{2 j-1}-1\right] \prod_{k=1}^{2 j-2} m_{k}\right)$ and $B^{\prime}=\varphi\left(\sum_{j=1}^{\rho^{\prime}}\left[0, m_{2 j}-1\right] \prod_{k=1}^{2 j-1} m_{k}\right)$.

Lemma 3.4 Suppose that $(A, B)$ is a 1-overlapped factorization of $\mathbb{Z}_{n}$ with $A=$ $\{0,1, \ldots, k-1\}$ for some $k \in \mathbb{Z}_{n}$. Then, $(A, B)$ is a DBNS 1-overlapped factorization.

Proof Suppose that there exists a 1-overlapped factorization of $\mathbb{Z}_{n}$ with $A=$ $\varphi(\{0,1, \ldots, k-1\})$ for some $k \in \mathbb{Z}$ which is not a DBNS 1-overlapped factorization.

Let us assume that $\varphi(0) \notin B$. Let $\varphi(b)$ be the doubly covered element of $(A, B)$. Then, we consider $(A, B+\varphi(b))$ instead of $(A, B)$ since shifting does not change the DBNS property. Thus, we can assume $\varphi(0) \in B$.

If each $\varphi(i k) \in B$ for each $i \in[1,(n+1-k) / k],(A, B)$ is a 1-overlapped factorizations. Thus we assume that there exists an integer $i \in[1,(n+1-k) / k]$ such that $\varphi(i k)$ does not belong to $B$. Let $i_{1}$ be the smallest one among such $i$. Let $\varphi(j)+\varphi\left(i_{1} k-j\right)$ be an expression of $\varphi\left(i_{1} k\right)$, where $j \in[2, k-1]$ and $\varphi\left(i_{1} k-j\right) \in B$. 
Then, we have

$$
\begin{aligned}
\varphi\left(i_{1} k-2\right) & =\varphi\left(\left(i_{1}-1\right) k\right)+\varphi(k-2) & \left(\varphi(k-2) \in A, \varphi\left(\left(i_{1}-1\right) k\right) \in B\right), \\
& =\varphi(j-2)+\varphi\left(i_{1} k-j\right) & \left(\varphi(j-2) \in A, \varphi\left(i_{1} k-j\right) \in B\right) . \\
\varphi\left(i_{1} k-1\right) & =\varphi\left(\left(i_{1}-1\right) k\right)+\varphi(k-1) & \left(\varphi(k-1) \in A, \varphi\left(\left(i_{1}-1\right) k\right) \in B\right), \\
& =\varphi(j-1)+\varphi\left(i_{1} k-j\right) & \left(\varphi(j-1) \in A, \varphi\left(i_{1} k-j\right) \in B\right) .
\end{aligned}
$$

These two equations contradict to the uniqueness of the doubly covered element. Therefore, $\varphi\left(i_{1} k\right)$ is factored as $\varphi(1)+\varphi\left(i_{1} k-1\right)$, where $\varphi(1) \in A$ and $\varphi\left(i_{1} k-1\right) \in B$. Hence the doubly covered element of $(A, B)$ is $\varphi\left(i_{1} k-1\right)$.

Suppose that there exist an integer $i \in\left[i_{1}+1,(n+1-k) / k\right]$ such that $\varphi(i k-1)$ does not belong to $B$ and let $i_{2}$ be the smallest one. If $\varphi\left(i_{2} k-1\right)$ is factored as $\varphi(j)+\varphi\left(i_{2} k-j-1\right)$, where $j \in[1, k-1]$ and $\varphi\left(i_{2} k-j-1\right) \in B, \varphi\left(i_{2} k-2\right)$ has two factorizations; $\left.\varphi\left(\left(i_{2}-1\right) k-1\right)\right)+\varphi(k-1)$ and $\varphi(j-1)+\varphi\left(i_{2} k-j-1\right)$. This contradicts the uniqueness of doubly covered element.

Consequently for each integer $i \in\left[i_{1}+1,(n+1-k) / k\right], \varphi(i k-1)$ belongs to $B$. Then, $\left(A, B-\varphi\left(i_{1} k-1\right)\right)$ is a basic DBNS factorization.

A subset $S$ of a cyclic group $\mathbb{Z}_{n}$ is $(a, i, k)$-arithmetic if $S=a+i[0, k-1]$. A subset $S$ of $\mathbb{Z}_{n}$ is $(i, k)$ quasi-arithmetic if $S$ is decomposable as $S=S^{\prime}+R$, where $S^{\prime}$ is $(a, i, k)$-arithmetic for some $a \in \mathbb{Z}_{n}, k \geq 2$, and $|S|=k|R|=\left|S^{\prime}\right| \cdot|R|$. A subset $S \subset \mathbb{Z}_{n}$ is quasi-arithmetic if $A$ is $(i, k)$ quasi-arithmetic for some $i \in \mathbb{Z}_{n}$ and $k \geq 2$.

Example 1 A symmetric subset $S \subset \mathbb{Z}_{n}$ is an arithmetic progression if $|S| \in\{2,3\}$. A subset $\{ \pm x, \pm y\} \subset \mathbb{Z}_{n}$ is quasi-arithmetic since $\{ \pm x, \pm y\}=\{x,-y\}+\{0, y-x\}$.

Example 2 Let $0, \pm x, \pm y$ be five distinct elements of $\mathbb{Z}_{n}$. Then, $\{0, \pm x, \pm y\}$ is quasiarithmetic if and only if one of the following holds;

(1) $\{ \pm x\}=\{ \pm 2 y\}$,

(2) $\{ \pm 2 x\}=\{ \pm y\}$.

Example 3 Let $\pm x, \pm y, \pm z$ be six distinct elements of $\mathbb{Z}_{n}$. If $x+y+2 z=0$, then $\{ \pm x, \pm y, \pm z\}$ is quasi-arithmetic since $\{ \pm x, \pm y, \pm z\}=\{x,-z,-x-2 z\}+\{0,2 z\}$. Let $\pm x, \pm y, \pm z, \pm w$ be eight distinct elements of $\mathbb{Z}_{n}$. If $x+y+z+w=0$, then $\{ \pm x, \pm y, \pm z, \pm w\}$ is quasi-arithmetic since $\{ \pm x, \pm y, \pm z, \pm w\}=\{x,-y\}+$ $\{0,-x-z\}+\{0, y+z\}$.

Lemma 3.5 Let $(A, B)$ be a 1-overlapped factorization of $\mathbb{Z}_{n}$. If $A$ is $(a, i, k)$ arithmetic, then both $i$ and $\varphi(k)$ are invertible elements of $\mathbb{Z}_{n}$ and $\left(i^{-1}(A-a), i^{-1} B\right)$ is a basic DBNS circulant factorization.

Proof From Theorem 2.1, there exists an integer $k^{\prime}$ such that $i k^{\prime}$ is a generator of $\mathbb{Z}_{n}$. Therefore, $i$ is an invertible element of $\mathbb{Z}_{n}$. Moreover, $\varphi(k)$ is also invertible since $n+1$ is a multiple of $k$.

Lemma 3.6 Let $(A, B)$ be a 1-overlapped factorization of $\mathbb{Z}_{n}$. Then, $(A, B)$ is a DBNS 1-overlapped factorization if and only if $A$ is quasi-arithmetic. 
Proof Define $A^{\prime}:=\{a, a+i, \ldots, a+(k-1) i\}$ and $A=A^{\prime}+C$. Then, $\left(A^{\prime}, B+C\right)$ is also a 1-overlapped factorization of $\mathbb{Z}_{n}$. Since $i$ is an invertible element of $\mathbb{Z}_{n}$ by Lemma 3.5, $\left(i^{-1}\left(A^{\prime}-a\right), i^{-1}(B+C)\right)$ is also a 1-overlapped factorization. From Lemma 3.4, there exists an element $z \in \mathbb{Z}_{n}$ such that $i^{-1}(B+C+z)=\varphi(k) \varphi([0,|B||C|-1])$ since we have $i^{-1}(A-a)=[0, k-1]$. Hence we have $i^{-1} \varphi(k)^{-1}(B+C+z)=[0,|B||C|-$ 1]. Then there exist $b, c \in \mathbb{Z}_{n}$ such that $b+c=z$ and $[0,|B||C|-1]$ contains both of $i^{-1} \varphi(k)^{-1}(B+b)$ and $i^{-1} \varphi(k)^{-1}(C+c)$. Then, for each $x \in i^{-1} \varphi(k)^{-1}(B+b)$ and $y \in i^{-1} \varphi(k)^{-1}(C+c)$, we obtain $0 \leq \psi(x)+\psi(y) \leq 2(|B||C|-1) \leq$ $\left|A^{\prime}\right||B||C|-2 \leq n-1$. Thus, there exist integers $\rho^{\prime}(\geq 1), m_{2}^{\prime}, m_{3}^{\prime}, \ldots, m_{2 \rho-1}^{\prime}(\geq 2)$ and $m_{1}^{\prime}, m_{2 \rho}^{\prime}(\geq 1)$ such that

$$
\begin{aligned}
i^{-1} \varphi(k)^{-1}(C+c) & =\sum_{j=1}^{\rho^{\prime}}\left[0, m_{2 j-1}^{\prime}-1\right] \prod_{l=1}^{2 j-2} m_{k}^{\prime}, \\
i^{-1} \varphi(k)^{-1}(B+b) & =\sum_{j=1}^{\rho^{\prime}}\left[0, m_{2 j}^{\prime}-1\right] \prod_{l=1}^{2 j-1} m_{k}^{\prime}, \text { and } \\
|B||C| & =\prod_{k=1}^{2 \rho^{\prime}} m_{l} .
\end{aligned}
$$

Notice that $i^{-1} \varphi(k)^{-1}\left(A^{\prime}-a\right)=\varphi(k)^{-1}[0, k-1]=\varphi\left([0, k-1] \prod_{l=1}^{2 \rho^{\prime}} m_{l}\right)$ since we have $k|B||C|=n+1$. Then, we obtain

$$
\begin{aligned}
i^{-1} \varphi(k)^{-1}(A+c-a) & =\varphi\left(\sum_{j=1}^{\rho^{\prime}+1}\left[0, m_{2 j-1}-1\right] \prod_{l=1}^{2 j-2} m_{2 j-1}\right), \\
i^{-1} \varphi(k)^{-1}(B+b) & =\varphi\left(\sum_{i=1}^{\rho^{\prime}}\left[0, m_{2 j}-1\right] \prod_{l=1}^{2 j-1} m_{2 j}\right) .
\end{aligned}
$$

From Lemma 2.5, $\left(i^{-1} \varphi(k)^{-1}(A+c-a), i^{-1} \varphi(k)^{-1}(B+b)\right)$ is a DBNS 1-overlapped factorization.

\section{Main results}

In this section, we prove that Conjecture 1.5 is true for every 1-overlapped factorization $(A, B)$ of $\mathbb{Z}_{n}$ with $\min \{|A|,|B|\} \leq 8$.

Lemma 4.1 Let $(A, B)$ be a 1-overlapped factorization of $\mathbb{Z}_{n}$. Then, there exist $g_{1}, g_{2} \in \mathbb{Z}_{n}$ such that $\left(A+g_{1}, B+g_{2}\right)$ is a symmetric 1-overlapped factorization with $0 \in A+a$ if and only if $|A|$ is odd.

Proof From Theorem 2.2, there exist $g_{1}^{\prime}, g_{2}^{\prime} \in \mathbb{Z}_{n}$ such that $\left(A+g_{1}^{\prime}, B+g_{2}^{\prime}\right)$ is a symmetric 1-overlapped factorization. If $|A|$ is odd, there exists an element $a \in A+g_{1}^{\prime}$ 
such that $2 a=0$ since $A+g_{1}^{\prime}$ is symmetric. If $a=0$, then $g_{1}:=g_{1}^{\prime}$ satisfies $0 \in A+g_{1}$. If $a \neq 0$, then $n$ is even, and $a=n / 2$. Then, $\left(A+g_{1}^{\prime}+a, B+g_{2}^{\prime}+a\right)$ is a symmetric 1-overlapped factorization, and $g_{1}=g_{1}^{\prime}+a$ satisfies $0 \in A+g_{1}$. Suppose that $|A|$ is even. Then, $n=|A| \cdot|B|-1$ is odd. For any $g_{1} \in \mathbb{Z}_{n}$ such that $A+g_{1}$ is symmetric, we have $0 \notin A+g_{1}$ since $\left|A+g_{1}\right|$ is even.

Lemma 4.2 Let $(A, B)$ be a 1-overlapped factorization of $\mathbb{Z}_{n}$. If A satisfies one of the following, then $A$ is quasi-arithmetic;

(1) $A=\{0, \pm x, \pm s\}$ and $\{0,2 x\}+2 s \subset B$,

(2) $A=\{ \pm x, \pm s, \pm t\}$ and $\{0,2 x\}+2 s+t \subset B$;

(3) $A=\{0, \pm x, \pm s, \pm t\}$ and $\{0,2 x\}+\{s+t,-x+s-2 t\} \subset B$;

(4) $A=\{ \pm x, \pm s, \pm t, \pm u\}$ and $\{0,2 x\}+s+t+u \subset B$.

Proof (1) Since $|(A+x+2 s) \cap B|=2$, we have $x+2 s=0$. Thus, $A$ is arithmetic as we showed in Example 2.

(2) Since $|(A+x+2 s+t) \cap B|=2$, we have $x+2 s+t=0$. Thus, $A$ is quasi-arithmetic as we showed in Example 3.

(3) From $\{0,2 x\}+s+t \subset(A+x+s+t) \cap B$ and (3.1), we have $x+s+t=0$. From $\{0,2 x\}+\{-x+s-2 t\} \subset(A+s-2 t) \cap B$ and (3.1), we have $s-2 t=0$. From these two relations, we have $A= \pm\{0, t, 2 t, 3 t\}$.

(4) Since $|(A+x+s+t+u) \cap B|=2$, we have $x+s+t+u=0$. Thus, $A$ is quasi-arithmetic as we showed in Example 3.

For a symmetric subset $S \subset \mathbb{Z}_{n}$ and an element $r \in S$, let $\Delta_{r}(S)$ be the set defined by

$$
\left\{a_{1}-a_{2} \mid a_{1}, a_{2} \in S, a_{1} \neq a_{2},\left\{a_{1}, a_{2}\right\} \neq\{ \pm r\}\right\}
$$

For subsets $S_{1}, S_{2} \subset \mathbb{Z}_{n}$ and elements $g_{1}, \ldots, g_{m}, h \in \mathbb{Z}_{n}$, define

$$
D_{S_{1}, S_{2}}^{h}\left(g_{1}, \ldots, g_{m}\right):=\left(S_{1}+h\right) \backslash\left(S_{2}+\left\{g_{1}, \ldots, g_{m}\right\}\right) .
$$

If $S_{3}=\left\{g_{1}, \ldots, g_{m}\right\}$, we write $D_{S_{1}, S_{2}}^{h}\left(S_{3}\right)$ instead of $D_{S_{1}, S_{2}}^{h}\left(g_{1}, \ldots, g_{m}\right)$.

Lemma 4.3 Suppose $A=\{ \pm x, \pm s, \pm t\}$. Let $D^{h}\left(b_{1}, \ldots, b_{m}\right)$ denote the set $D_{A, \Delta_{x}(A)}^{h}\left(b_{1}, \ldots, b_{m}\right)$.

Then,

$$
\begin{aligned}
& D^{s+t}(x,-x) \subset\{2 s+t, 2 t+s\}, \\
& D^{s-t}(x,-x, 2 t+s) \subset\{2 s-t\}, \\
& D^{x+3 t}(2 t+s) \backslash\{x+4 t\} \subset\{2 x+3 t, x+3 t-s\} . \\
& 2 x+(2 t-s) \in\left(\Delta_{x}(A)+2 x+3 t\right) \cap\left(\Delta_{x}(A)+x+3 t-s\right), \\
& D^{2 x+s+t}(x) \backslash(\{3 x+s+t\} \cup\{2 x+2 s+t\}) \subset\{2 x+s+2 t\}, \\
& D^{2 x+\gamma(t-s)}(x, 3 x+s+t) \backslash\{2 x+\gamma(2 t-s)\} \subset\{2 x-\gamma(2 s-t)\},(\gamma \in\{ \pm 1\}) .
\end{aligned}
$$


Proof By using

$$
\Delta_{x}(A)=\{ \pm x \pm s, \pm x \pm t, \pm s \pm t, \pm 2 s, \pm 2 t\}
$$

these containment relations can be checked directly.

Lemma 4.4 Suppose that $A=\{0, \pm x, \pm s, \pm t\}$. Let $D^{h}\left(b_{1}, \ldots, b_{m}\right)$ denote the set $D_{A, \Delta_{x}(A)}^{h}\left(b_{1}, \ldots, b_{m}\right)$. Then,

$$
\begin{aligned}
& D^{s+t}(x,-x) \backslash\{s+t\} \subset\{2 s+t, 2 t+s\}, \\
& D^{s-t}(x,-x, s+t) \subset\{2 s-t,-2 t+s\}, \\
& D^{s}(x,-x, 2 s-t) \subset\{s+t\}, \\
& D^{2 t}(x,-x, s+t,-2 s+t) \subset\{3 t\}, \\
& D^{x-2 t}(x,-(s+t),-3 t, 2 x+s-t)=\emptyset, \\
& D^{x+s-t}(x, 2 s-t) \backslash\{2 x+s-t\} \subset\{x+(s-2 t)\}, \\
& D^{2 x+s}(x, x-s+2 t) \backslash\{3 x+t\} \subset\{2 x+s+t\} .
\end{aligned}
$$

Proof By using

$$
\Delta_{x}(A)=\{ \pm x, \pm s, \pm t, \pm 2 s, \pm 2 t, \pm x \pm s, \pm x \pm t, \pm s \pm t\}
$$

these containment relations can be checked directly.

Lemma 4.5 Suppose that $A=\{ \pm x, \pm s, \pm t, \pm u\}$. Let $D^{h}\left(b_{1}, \ldots, b_{m}\right)$ denote the set $D_{A, \Delta_{x}(A)}^{h}\left(b_{1}, \ldots, b_{m}\right)$. Then,

$$
\begin{aligned}
& D^{s-t}(x,-x) \backslash\{s-t+u, s-t-u\} \subset\{2 s-t,-2 t+s\}, \\
& D^{s+t}(x,-x, s-t+u,-(s-t+u)) \subset\{2 s+t, 2 t+s\}, \\
& D^{s+t}(x,-x, 2 s-t) \subset\{s+t+u, s+t-u\}, \\
& D^{s-u}(x,-x, 2 s-t, s+t+u) \subset\{s-2 u\}, \\
& D^{x+s-t+u}(x,-2 u+s, 2 t-u) \subset\{x+2 s+t-u, 2 x+s+t-u\}, \\
& D^{x-s-t+u}(x,-2 t+u, 2 u-s, 2 x+u+s-t) \subset\{x-2 s-t+u\}, \\
& D^{2 x+u-s}(x, x-2 s-t+u, x+2 t+u-s) \subset\{2 x+2 u-s, 3 x+u-s\}, \\
& D^{2 x-s+t}(x, x+2 t+u-s, 2 x+s+t-u, 3 x-s+u) \subset\{2 x-2 s+t\}, \\
& D^{x+2 t-s}(2 x+s+t-u, 2 s-t,-2 u+s) \subset\{x+3 s-u\}, \\
& D^{x+s+2 u}(x, 2 x+s+t-u, s+t+u, x+3 s-u) \subset\{x+2 s+2 t\}, \\
& D^{3 s+t}(x+2 s+2 t, s+t+u, 2 s-t,-x+2 s+t-u) \subset\{4 s+t\}, \\
& D^{3 s+u}(x+2 s+2 u, 2 s-t, 4 s+t) \subset\{-x+3 s+u\}, \\
& D^{2 x-u-s}(x, x-3 s-u, 2 x-s+t+u, x-2 u-s+t) \backslash\{3 x-u-s\}
\end{aligned}
$$




$$
\begin{aligned}
& \subset\{2 x-s-t-u\}, \\
& D^{2 x-s-t}(x, x-3 t-s, 2 x-s+t+u, x-2 s-t+u, 3 x-u-s) \\
& \subset\{2 x-s-t-u\} .
\end{aligned}
$$

Proof By using

$$
\Delta_{x}(A)=\{ \pm 2 s, \pm 2 t, \pm 2 u, \pm x \pm s, \pm x \pm t, \pm x \pm u, \pm s \pm t, \pm t \pm u, \pm u \pm s\},
$$

these containment relations can be checked directly.

Lemma 4.6 Let $(A, B)$ be a symmetric 1-overlapped factorization of $\mathbb{Z}_{n}$, and $x$ an element of $A \cap B$ which satisfies (3.2). Then, we have $\left(\Delta_{x}(A)+B\right) \cap B=\emptyset$.

Proof From Lemma 3.1, $\Delta_{x}(A) \cap(B-b)=\emptyset$ for any $b \in B$.

Lemma 4.7 Let $(A, B)$ be a symmetric 1-overlapped factorization of $\mathbb{Z}_{n}$, and $x$ an element of $A \cap B$ which satisfies (3.2). Then, for any $B_{0} \subset B$ and $i \in \mathbb{Z}_{n}$, we have $D_{A, \Delta_{x}(A)}^{i}\left(B_{0}\right) \cap B \neq \emptyset$. In particular, $D_{A, \Delta_{x}(A)}^{i}\left(B_{0}\right) \subset\left\{b_{0}\right\}$ yields $b_{0} \in B$.

Proof From Lemma 4.6, $\varnothing \neq(A+i) \cap B \subset(A+i) \backslash\left(\Delta_{x}(A)+B\right) \subset D_{A, \Delta_{x}(A)}^{i}\left(B_{0}\right)$.

Although Lemma 4.7 will be frequently used in the proof of Theorem 4.10, we will not mention it.

Lemma 4.8 Let $(A, B)$ be a symmetric 1-overlapped factorization of $\mathbb{Z}_{n}$ such that $(A-A) \cap(B-B)=\{0, \pm 2 x\}$. Let $s \in \mathbb{Z}_{n}$ be an element such that $2 s \in \Delta_{x}(A)$. Then, for any $i \in \mathbb{Z}_{n}$, there exists $\alpha \in\{ \pm 1\}$ such that $i+\alpha s \notin B$.

Proof Since otherwise, we have $2 s \in \Delta_{x}(A) \cap(B-B)$, which contradicts Lemma 3.1 .

Lemma 4.9 Let $(A, B)$ be a symmetric 1-overlapped factorization of $\mathbb{Z}_{n}$ such that $(A-A) \cap(B-B)=\{0, \pm 2 x\}$, and that $\{ \pm x\}+c \subset B$. Then $c=0$.

Proof This lemma follows from $|(A+c) \cap B| \geq 2$ and (3.1).

Theorem 4.10 A 1-overlapped factorization $(A, B)$ of $\mathbb{Z}_{n}$ is a DBNS 1-overlapped factorization if $\min \{|A|,|B|\} \leq 8$.

Proof Without loss of generality, we can assume $|A| \leq|B|$. From Theorem 2.2, we can assume that $(A, B)$ is a symmetric 1-overlapped factorization. From Lemma 3.6, all we have to prove is that $A$ is quasi-arithmetic. As we saw in Example 1, a symmetric set $A$ is quasi-arithmetic if $|A| \leq 4$. Thus, we assume $5 \leq|A| \leq 8$. From Lemma 3.1, there exists a unique nonzero element $x \in A \cap B$ up to sign such that

$$
(A-A) \cap(B-B)=\{0, \pm 2 x\} .
$$


First, we prove the case $|A|=5$. From Lemma 4.1, we can assume that $A=$ $\{0, \pm x, \pm y\}$. Then we have

$$
\Delta_{x}(A)=\{ \pm x, \pm y, \pm x \pm y, \pm 2 y\}
$$

Let us define $D_{A, \Delta_{x}(A)}^{a}\left(b_{1}, \ldots, b_{m}\right)=D^{a}\left(b_{1}, \ldots, b_{m}\right)$. Then, the following containment relations can be checked directly.

$$
\begin{aligned}
D^{2 x+y}(x) \backslash\{3 x+y\} & \subset\{2 x+2 y\}, \\
D^{2 x-y}(x, 3 x+y) & \subset\{2 x-2 y\}, \\
D^{y}(x,-x) & \subset\{2 y\},
\end{aligned}
$$

From (4.29) and (4.30), one of $2 x+2 y \in B$ and $2 x-2 y \in B$ holds. From (4.31), we obtain $\{ \pm 2 y\} \subset B$. Then from Lemma 4.2 (1), $A$ is quasi-arithmetic.

Next, we prove the case $|A|=6$.

From Theorem 2.2 and (4.1), there exist $y, z \in \mathbb{Z}_{n}$ such that $A=\{ \pm x, \pm y, \pm z\}$, and that

$$
\{ \pm(y+2 z)\} \subset B
$$

Then, from (4.32), (4.2) with $(s, t)=(y, z)$ yields

$$
\{ \pm(2 y-z)\} \subset B
$$

We claim that there exists $\alpha \in\{ \pm 1\}$ such that $x+4 \alpha y \notin B$. Indeed, otherwise, the symmetry of $B$ yields $(A+4 y) \cap B \supset\{ \pm x+4 y\}$, i.e., $4 y=0$ from Lemma 4.9. Note that 4 is an invertible element since $n+1$ is a multiple of 6 . Thus, we have $y=0$, which contradicts $|A|=6$. By the same reason, there exists also $\beta \in\{ \pm 1\}$ such that $x+4 \beta z \notin B$.

From (4.5) with $(s, t)=(\alpha y, \alpha z), 3 x+\alpha(y+z) \notin B$ yields $2 x+\alpha(y+2 z) \in B$. From (4.6) with $(\gamma, s, t)=(\alpha \beta, \alpha y, \alpha z), 3 x+\alpha(y+B) \in B$ yields $2 x-\beta(2 y-z) \in B$. Thus, one of $2 x+\alpha(y+2 z) \in B$ and $2 x-\beta(2 y-z) \in B$ holds. In the former case, $A$ is quasi-arithmetic by (4.32) and Lemma 4.2 (2). In the latter case, the same holds by (4.33) and Lemma 4.2 (2).

Next, we prove the case $|A|=7$. From Lemma 4.1, (4.7), and (4.8), there exist $y, z$ such that $A=\{0, \pm x, \pm y, \pm z\}$, and that

$$
\{ \pm(2 y-z)\} \subset B
$$

Then, from (4.34), (4.9) with $(s, t)=(y, z)$ yields

$$
\{ \pm(y+z)\} \subset B
$$

From (4.34) and (4.35), (4.10) with $(s, t)=(y, z)$ yields

$$
\{ \pm 3 z\} \subset B .
$$


We claim

$$
2 x \pm(y-z) \notin B \text {. }
$$

Indeed, if $2 x+\alpha(y-z) \in B$ for some $\alpha \in\{ \pm 1\}$, then (4.35), (4.36), and (4.11) with $(s, t)=(\alpha y, \alpha z)$ yield a contradiction to Lemma 4.7. From (4.34) and (4.37), (4.12) with $(s, t)= \pm(y, z)$ yields

$$
\{ \pm x \pm(y-2 z)\} \subset B
$$

Note that there exists $\beta \in\{ \pm 1\}$ such that $3 x+\beta z \notin B$, from Lemma 4.8. Then from (4.38), (4.13) with $(s, t)=(\beta y, \beta z)$ yields

$$
2 x+\beta(y+z) \in B .
$$

Then from Lemma 4.2 (3) with $(s, t)=(\beta y, \beta z),(4.35),(4.38)$, and (4.39), $A$ is quasi-arithmetic.

Finally, we prove the case $|A|=8$. From Theorem 2.2, (4.14), and (4.15), there exist $x, y_{1}, y_{2} \in \mathbb{Z}_{n}$ such that $\left\{ \pm x, \pm y_{1}, \pm y_{2}\right\} \subset A$, and that

$$
\left\{ \pm\left(2 y_{1}-y_{2}\right)\right\} \subset B
$$

From (4.40) and (4.16) with $(s, t)=\left(y_{1}, y_{2}\right)$, there exists $y_{3} \in A$ distinct from $\pm x, \pm y_{1}, \pm y_{2}$ such that

$$
\left\{ \pm\left(y_{1}+y_{2}+y_{3}\right)\right\} \subset B
$$

From (4.40) and (4.41), (4.17) with $(s, t, u)=\left(y_{1}, y_{2}, y_{3}\right)$ yields

$$
\left\{ \pm\left(2 y_{3}-y_{1}\right)\right\} \subset B
$$

From (4.41) and (4.42), (4.17) with $(s, t, u)=\left(y_{3}, y_{1}, y_{2}\right)$ yields

$$
\left\{ \pm\left(2 y_{2}-y_{3}\right)\right\} \subset B
$$

From (4.18), (4.40), (4.42), and (4.43),

$$
\left\{x+\alpha\left(2 y_{j}+y_{j+1}-y_{j+2}\right), 2 x+\alpha\left(y_{j}+y_{j+1}-y_{j+2}\right)\right\} \cap B \neq \emptyset
$$

for all $\alpha \in\{ \pm 1\}$ and $j \in\{1,2,3\}$, where indices are read modulo 3. For $i \in \mathbb{Z}_{3}$, let $S_{i}$ denote the set $\left\{x \pm\left(2 y_{j}+y_{j+1}-y_{j+2}\right)\right\}$.

Claim If B contains at least two sets among $S_{1}, S_{2}$, and $S_{3}$, then $A$ is quasi-arithmetic. 
Proof Suppose that $S_{i} \cup S_{i+1} \subset B$. Since $B$ is symmetric, $\{ \pm x\}+\left\{2 y_{i}+y_{i+1}-\right.$ $\left.y_{i+2}, 2 y_{i+1}+y_{i+2}-y_{i}\right\} \subset B$. Then, from Lemma 4.9, we have

$$
\left\{\begin{array}{l}
2 y_{i}+y_{i+1}-y_{i+2}=0 \\
2 y_{i+1}+y_{i+2}-y_{i}=0
\end{array}\right.
$$

Solving these equations, we obtain

$$
y_{i}=-3 y_{i+1}, \quad y_{i+2}=-5 y_{i+1} .
$$

Note that there exists $\alpha \in\{ \pm 1\}$ such that $3 x+2 \alpha y_{i+1} \notin B$ from $4 y_{i+1}=y_{i+1}-y_{i} \in$ $\Delta_{x}(A)$ and Lemma 4.8. By using (4.45), we have

$$
D^{2 x+2 \alpha y_{i+1}}(x) \backslash\left\{3 x+2 \alpha y_{i+1}\right\} \subset\left\{2 x \pm 7 y_{i+1}\right\}=\left\{2 x \pm\left(y_{1}+y_{2}+y_{3}\right)\right\} .
$$

Thus, there exists $\beta \in\{ \pm 1\}$ such that $2 x+\beta\left(y_{1}+y_{2}+y_{3}\right) \in B$. From Lemma 4.2 (4) and (4.41), $A$ is quasi-arithmetic.

Hence, we consider the case where $B$ contains at most one among $S_{1}, S_{2}, S_{3}$. Suppose that

$$
\begin{aligned}
& \left\{x \pm\left(2 y_{i}+y_{i+1}-y_{i+2}\right)\right\} \not \subset B, \\
& \left\{x \pm\left(2 y_{i+1}+y_{i+2}-y_{i}\right)\right\} \not \subset B .
\end{aligned}
$$

From (4.46), there exists $\alpha \in\{ \pm 1\}$ such that

$$
x+\alpha\left(2 y_{i}+y_{i+1}-y_{i+2}\right) \notin B .
$$

Define $(w, y, z):=\left(\alpha y_{i}, \alpha y_{j+1}, \alpha y_{j+2}\right)$. Then, from (4.48), (4.44) with $j=i$ yields

$$
\{ \pm(2 x+w+y-z)\} \subset B
$$

Moreover, (4.40), (4.41), (4.42), and (4.43) yield

$$
\{ \pm(2 y-z), \pm(2 z-w), \pm(2 w-y), \pm(y+z+w)\} \subset B
$$

From (4.49) and (4.50), (4.19) with $(s, t, u)=(y, z, w)$ yields

$$
\{ \pm(x-2 y-z+w)\} \subset B
$$

From (4.47) and (4.51), we have $x+(2 y+z-w) \notin B$. Then, (4.44) with $j=i+1$ yields

$$
\{ \pm(2 x+y+z-w)\} \subset B
$$


From (4.50) and (4.52), (4.19) with $(s, t, u)=(z, w, y)$ yields

$$
\{ \pm(x-2 z-w+y)\} \subset B
$$

Here, we consider two cases.

(Case 1) Suppose that

$$
x+2 z+w-y \in B
$$

Then, (4.53) and (4.54) yield $\{ \pm x+2 z+w-y\} \subset B$. From Lemma 4.9, we have

$$
2 z+w-y=0
$$

From (4.51) and (4.54), (4.20) with $(s, t, u)=(y, z, w)$ yields one of

$$
\begin{gathered}
2 x+2 w-y \in B, \\
3 x+w-y \in B .
\end{gathered}
$$

Suppose that $2 x+2 w-y \in B$. Then, from (4.50) and Lemma 4.9, we have

$$
x+2 w-y=0 .
$$

By using (4.55) and (4.57), we have $2 x+(w+y-z)=(x-w)+(y+z+$ $w) \in \Delta_{x}(A)+(y+z+w)$, which contradicts (4.49), (4.50), and Lemma 4.6. Thus, (4.56) holds. From (4.52), (4.54), and (4.56), (4.21) with $(s, t, u)=(y, z, w)$ yields $2 x-2 y+z \in B$. Then, from (4.50) and Lemma 4.9, we have

$$
x-2 y+z=0
$$

Then, from (4.55), $x-(y+z+w)=0$. Therefore, $A$ is quasi-arithmetic as we saw in Example 3.

(Case 2) Suppose that $x+2 z+w-y \notin B$. Then, (4.44) with $j=i+2$ yields

$$
\{ \pm(2 x+z+w-y)\} \subset B
$$

From (4.50) and (4.58), (4.19) with $(s, t, u)=(w, y, z)$ yields

$$
\{ \pm(x-2 w-y+z))\} \subset B .
$$

From (4.49), (4.50), (4.52), and (4.58), (4.22) where $(s, t, u)$ is a cyclic permutation of $(w, y, z)$ yields

$$
\{ \pm(x+3 w-z), \pm(x+3 y-w), \pm(x+3 z-y)\} \subset B .
$$


Then, from (4.49), (4.50), (4.52), and (4.58), (4.23) where $(s, t, u)$ is a cyclic permutation of $(w, y, z)$ yields

$$
\{x+2 y+2 z, x+2 z+2 w, x+2 w+2 y\} \subset B .
$$

From (4.50), (4.51), (4.53) and (4.60), (4.24) with $(s, t, u)=(y, z, w)$ and $(s, t, u)=$ $(z, w, y)$ yields

$$
\{4 y+z, 4 z+w\} \subset B
$$

Then, from (4.50) and (4.60), (4.25) with $(s, t, u)=(y, z, w)$ and $(s, t, u)=(z, w, y)$ yields

$$
\{ \pm(x-3 y-w), \pm(x-3 z-y)\} \subset B .
$$

From (4.51), (4.58), and (4.59), (4.26) and (4.27) with $(s, t, u)=(y, z, w)$ yield $2 x-(y+z+w) \in B$. From Lemma 4.2 (4) and (4.50), $A$ is quasi-arithmetic.

\section{Concluding remarks}

In [20], one of the authors determined all minimally non-ideal DBNS Lehman matrices. If Conjecture 1.5 is true, we will be able to determine the shapes of circulant thin minimally non-ideal matrices. One of the related important open problems is the following.

Problem 5.1 Does there exist a DBNS Lehman matrix such that it is not ideal, but it is the core of an ideal matrix?

It is well known that projective planes over a finite field also produce circulant fat Lehman matrices. Characterization of circulant fat Lehman matrices is also an important problem.

Acknowledgments The authors would like to thank an editor and a referee for very useful comments which helped us to improve the paper substantially.

\section{References}

1. Bridges, W.G., Ryser, H.J.: Combinatorial designs and related systems. J. Algebra 13, 432-436 (1969)

2. Chudnovsky, M., Robertson, N., Seymour, P., Thomas, R.: The strong perfect graph theorem. Ann. Math. 164, 51-229 (2006)

3. Chvatal, V., Graham, R.L., Perold, A.F., Whitesides, S.H.: Combinatorial designs related to the perfect graph conjecture. Ann. Discret. Math. 21, 197-204 (1984)

4. Cornuéjols, G.: Combinatorial Optimization. Packing and Covering. CBMS-NSF Regional Conference Series in Applied Mathematics, vol. 74. Society for Industrial and Applied Mathematics (SIAM), Philadelphia (2001)

5. Cornuéjols, G., Guenin, B., Tunçel, L.: Lehman matrices. J. Comb. Theory Ser. B 99, 531-556 (2009)

6. Cornuéjols, G., Novick, B.: Ideal 0, 1 matrices. J. Comb. Theory Ser. B 60, 145-157 (1994)

7. De Bruijn, N.G.: On number systems. Nieuw Archief voor Wiskd. 3, 15-17 (1956) 
8. De Caen, D., Gregory, D.A., Hughes, I.G., Kreher, D.L.: Near-factors of finite groups. Ars Comb. 29, 53-63 (1990)

9. Fulkerson, D.R.: Blocking and anti-blocking pairs of polyhedra. Math. Program. 1, 168-194 (1971)

10. Gasparyan, G., Preissmann, M., Sebő, A.: Imperfect and nonideal clutters: a common approach. Combinatorica 23, 283-302 (2003)

11. Grinstead, C.M.: On circular critical graphs. Discret. Math. 51, 11-24 (1984)

12. Kashiwabara, K., Sakuma, T.: Grinstead's conjecture is true for graphs with a small clique number. Discret. Math. 306, 2572-2581 (2006)

13. Lehman, A.: Width-length inequality. Math. Program. 17, 403-417 (1979)

14. Lütolf, C., Margot, F.: A catalog of minimally nonideal matrices. Math. Methods Oper. Res. 47, 221241 (1998)

15. Padberg, M.: Perfect zero-one matrices. Math. Program. 6, 180-196 (1974)

16. Sachs, H.: On the Berge conjecture concerning perfect graphs. In: Guy, R., Hanani, H., Sauer, N., Schonheim, J. (eds.) Combinatorial Structures and their Applications. Proceedings of the Calgary International Conference on Combinatorial Structures and their Applications, pp. 377-384. Gordon and Breach, New York (1970)

17. Seymour, P.: The forbidden minors of binary clutters. J. Lond. Math. Soc. (2) 12, 356-360 (1976)

18. Shepherd, F.B.: Applying Lehman's theorems to packing problems. Math. Program. 71, 353-367 (1995)

19. Shinohara, H.: Thin Lehman matrices arising from finite groups. Linear Algebra Appl. 436, 850-857 (2012)

20. Shinohara, H.: On circulant non-ideal clutters (in press).

21. Szabó, S., Sands, A.D.: Factoring Groups into Subsets. CRC Press, Boca Raton (2009)

22. Wang, J.: Thin Lehman matrices and their graphs. Electron. J. Comb. 17(1), Research Paper 165 (2010)

23. Wang, J.: A new infinite family of minimally nonideal matrices. J. Comb. Theory Ser. A 118, 365-372 (2011) 